\title{
ESPECIES DE MACROMICETOS CITADAS DE MEXICO IX. ASCOMYCETES, PARTE III: 1983-1996
}

\author{
Rosario Medel \\ GASTON GuZMAN \\ Y \\ SANTIAgo ChACON \\ Instituto de Ecología, A.C. \\ Apartado postal 63 \\ 91000 Xalapa, Veracruz
}

\begin{abstract}
RESUMEN
Se presenta el tercer listado de los ascomicetos macroscópicos conocidos en México hasta 1996. Se han registrado a la fecha 544 especies para el país, de las cuales 269 pertenecen al período 1983-1996. Se discute la distribución ecológica y geográfica de las especies y se hace un análisis del desarrollo del conocimiento de estos organismos en México.
\end{abstract}

\section{ABSTRACT}

The third check-list of macroscopic ascomycetes known in Mexico until 1996 is presented. There are 544 species recorded from Mexico, of which 269 were reported between 1983-1996. The ecological and geographical distribution of the species is discussed and a brief analysis of the development of the knowledge of these fungi in Mexico is made.

\section{INTRODUCCION}

Los ascomicetos son el grupo de hongos más grande dentro del reino Fungi, ya que según Hawksworth et al. (1995) existen más de 32000 especies a nivel mundial, en comparación con las 22000 especies de basidiomicetos y las 700 y 1000 de cada uno de los demás grupos. En México estos organismos han sido poco estudiados y según los listados de García-Romero et al. (1970) y Chacón y Guzmán (1983a), se conocían 261 especies de ascomicetos macroscópicos hasta 1982. De acuerdo con el criterio de Hawksworth (1993), la primera herramienta en la documentación de la micobiota de una región, son las listas de las especies que ya se han registrado junto con su bibliografía asociada. De esta manera, el presente trabajo constituye, como los anteriores de la serie, una base para el conocimiento de los ascomicetos en México y es una recopilación de lo registrado hasta ahora. Se integra así el tercer listado de ascomicetos macroscópicos 
registrados para México, en donde se adicionan 269 especies a las ya conocidas y se incluyen además 14 no consideradas en los listados anteriores. Se hace un breve análisis de los grupos taxonómicos a los que pertenecen estos hongosy de la distribución ecológica y geográfica de las especies, además del desarrollo del conocimiento general del grupo.

\section{MATERIALES Y METODOS}

Se revisó exhaustivamente toda la bibliografía disponible a partir de 1983, pero se consideraron también cuatro citas anteriores a esta fecha que no se habían incluido en los dos listados pasados. En este trabajo se siguió el mismo criterio que en ambas enumeraciones anteriores, por lo que no se tomaron en cuenta taxa citados en memorias o resúmenes de congresos y de symposia o tesis. Se actualizó la clasificación empleada en los listados de 1970 y 1983, siguiendo en general el criterio de Hawksworth et al. (1995). Siempre que fue posible se pusieron al día también los nombres de las especies, pero se anotaron los sinónimos respectivos. Con respecto a los tipos de vegetación señalados en el listado, se trató de interpretar éstos lo mejor posible, según la información bibliográfica disponible de las localidades referidas. Se consideran cinco categorías de cubierta vegetal, a saber: vegetación tropical (incluye los bosques tropical perennifolio, caducifolio y subperennifolio y/o selvas baja, mediana y alta, así como los correspondientes acahuales y potreros) y bosques mesófilo de montaña, de coníferas, de pino-encino y de encino, que son los principales ecosistemas en donde crecen los ascomicetos en el país.

\section{RESULTADOS}

Se encontraron 59 referencias bibliográficas de los años 1983-1996 que citan ascomicetos macroscópicos de México, las cuales registran 269 especies. Se toman en cuenta además cuatro referencias no consideradas anteriormente en los dos listados publicados de esta serie en los que se mencionan 14 más. Las 269 del período 1983-1996, junto con las 261 registradas previamente, más las 14 adicionales, hacen un total de 544 especies conocidas en México. En el Cuadro 1 se presentan las descubiertas entre 19831996, señalando las 14 que pertenecen a los períodos anteriores; en todas se anota la entidad federativa de donde se citaron, el tipo de vegetación (cuando la información fue disponible) y la referencia bibliográfica en la que se citó por primera vez el hongo, de acuerdo con la clave del Cuadro 2.

Las 544 especies de ascomicetos registradas de México se adscriben a 10 órdenes, como se observa en el Cuadro 3. De éstos, los Xylariales y los Pezizales son los mejor representados con 243 y 127 especies respectivamente; les siguen en importancia los Helotiales y Sordariales con 56 y 44, y los grupos menos conocidos son los Diaporthales y Ostropales con no más de 2 cada uno. En el mencionado Cuadro 3 se anota el número de especies en los períodos en los que fueron registradas. 
Cuadro 1. Especies de ascomicetos macroscópicos citadas de MÚxico de 1983 a 1996*.

\begin{tabular}{|c|c|c|}
\hline Especies & Entidades & Literatura \\
\hline Anthostomella cf. melanosticta Ellis \& Everh. & Tamps. (VT) & 57 \\
\hline Arachnopeziza aurelia (Pers.: Fr.) Fuckel & Son. (BE) & 45 \\
\hline Ascodesmis macrospora W. Obrist. & D.F. & 44 \\
\hline Balansia cyperi Edgertom & Ver. & 4 \\
\hline Belenyoscypha sessilis (Desm.) Dennis & Mor. (BM) & 7 \\
\hline Biscogniauxia atropunctata (Schwein.) Pouzar & N.L. (BE) & 54 \\
\hline B. divergens (Theiss.) Whalley \& Laessøe & Chis. (VT) & 54 \\
\hline $\begin{array}{l}\text { B. divergens var. macrospora (J. H. Mill.) Whalley \& } \\
\text { Laessøe apud Whalley, Laessøe \& Kile } \\
\text { - = Hypoxylon divergens var. macrospora J. H. Mill. }\end{array}$ & sin loc. & 39 \\
\hline B. grenadensis (J. H. Mill.) Whalley \& Laessøe & Chis. (BE, VT) & 54 \\
\hline $\begin{array}{l}\text { B. mediterranea (De Not.) Kuntze } \\
=\text { Hypoxylon mediterraneum (De Not.) J. H. Mill. }\end{array}$ & N.L., Tamps. (BM) & 54 \\
\hline B. nummularia var. exutans (Cooke) van der Gaucht & N.L., Tamps. (BC) & 54 \\
\hline B. nummularia var. merrilli (Bres.) van der Gaucht & $\begin{array}{l}\text { Camp., Q. Roo, } \\
\text { Tamps. (VT) }\end{array}$ & 54 \\
\hline B. cf. nummularia var. nummularia (Bull.: Fr.) Kuntze & Chis. (BPE) & 54 \\
\hline $\begin{array}{l}\text { B. nummularia var. pseudopachyloma San Martín \& } \\
\text { J. D. Rogers }\end{array}$ & $\begin{array}{l}\text { Chis., N.L., Tamps. } \\
\text { (VT) }\end{array}$ & 54 \\
\hline Bulgaria inquinans $\mathrm{Fr}$. & Mor. (BM) & 2 \\
\hline Calycella sulfurina (Quél.) Boud. & Mor. (BM) & 7 \\
\hline Calyculosphaeria calyculus (Mont.) Fitz. & Chis., Q. Roo (VT) & 50 \\
\hline C. tetraspora (Nannf.) San Martín & Chis. (VT) & 50 \\
\hline Camillea cyclisca (Mont.) Laessøe & Chis., Tamps. (VT) & 54 \\
\hline C. fossulata (Mont.) Laessøe, J. D. Rogers \& Whalley & Chis. (VT) & 54 \\
\hline $\begin{array}{l}\text { C. cf. gigaspora (Massee) Laessøe, J. D. Rogers \& } \\
\text { Whalley }\end{array}$ & Chis. (VT) & 54 \\
\hline $\begin{array}{l}\text { C. glycyrhiza (Berk. \& M. A. Curtis) Laessøe, J. D. } \\
\text { Rogers \& Whalley } \\
\text { - = Hypoxylon glycyrrhiza Berk. \& M. A. Curtis }\end{array}$ & sin loc. & 39 \\
\hline C. guzmanii San Martín \& J. D. Rogers & Ver. (VT) & 54 \\
\hline $\begin{array}{l}\text { C. harnesii (J. D. Rogers \& Dumont) Laessøe, J. D. } \\
\text { Rogers \& Whalley }\end{array}$ & Chis., Oax. (VT) & 54 \\
\hline $\begin{array}{l}\text { C. heterostoma var. macrospora (J. H. Mill.) Laessøe, } \\
\text { J. D. Rogers \& Whalley } \\
=\text { H. heterostomun var. macrospora J. H. Mill. }\end{array}$ & Ver. (VT) & 40 \\
\hline C. labellum Mont. & $\begin{array}{l}\text { Camp., Chis., Tamps., } \\
\text { Ver. (VT) }\end{array}$ & 37 \\
\hline C. magnifica San Martín \& J. D. Rogers & Tamps. (VT) & 54 \\
\hline C. mexicana San Martín \& J. D. Rogers & Tamps. (VT) & 54 \\
\hline C. mucronata Mont. & Chis. (VT) & 33 \\
\hline C. obularia (Fr.) Laessøe, J. D. Rogers \& Lodge & Oax., Tamps. (VT) & 54 \\
\hline $\begin{array}{l}\text { C. punctulata (Berk. \& Ravenel) Laessøe, J. D. Rogers } \\
\text { \& Whalley } \\
=\text { Hypoxylon punctulatum (Berk. \& Ravenel) Cooke }\end{array}$ & Chis., N.L. (BM, BPE) & 35,54 \\
\hline C. stellata Laessøe, J. D. Rogers \& Whalley & Chis., Q. Roo (VT) & 54 \\
\hline
\end{tabular}


Cuadro 1. Continuación.

\begin{tabular}{|c|c|c|}
\hline Especies & Entidades & Literatura \\
\hline C. tinctor (Berk.) Laessøe & $\begin{array}{l}\text { Chis., N.L., Tamps., } \\
\text { Ver. (BM, VT) }\end{array}$ & 54 \\
\hline C. cf. verruculospora J. D. Rogers, Laessøe \& Lodge & Chis. (VT) & 54 \\
\hline Cheilymenia coprinaria (Cooke) Boud. & Mor. (BC) & 9 \\
\hline Ch. crucipila (Cooke \& Phillips) Le Gal & Edo. Méx. (BC) & 11 \\
\hline Ch. rubra (Cooke) Boud. & Ver. $(B C)$ & 11 \\
\hline $\begin{array}{l}\text { Chlorociboria aeruginosa (Pers. per Pers.) Seaver ex } \\
\text { Ram., Korf \& Batch. }\end{array}$ & $\begin{array}{l}\text { Coah., Edo. Méx., } \\
\text { Hgo., Qro., Ver. } \\
\text { (BC, BPE, BM) }\end{array}$ & 58 \\
\hline Chloroscypha sabinae (Fuckel) Dennis & Mor. (BPE) & 3 \\
\hline Ciboria rufo-fusca (Weberb.) Sacc. & Edo. Méx. (BC) & 7 \\
\hline Claussenomyces salviicolor (Ellis \& Everh.) Korf \& Dixon & Ver. (BM) & 22 \\
\hline Cordyceps gracilis Mont. \& Durieu & Jal. (BM) & 47 \\
\hline Corynectes globosus (Sommerf.) Durand & Chis. (BC) & 7 \\
\hline $\begin{array}{l}\text { Creosphaeria sassafras (Schwein.: Fr.) Y. M. Ju, San } \\
\text { Martín \& J. D. Rogers }\end{array}$ & Tamps. (VT) & 57 \\
\hline Cudonia circinans (Pers.) Fr. & Mich. (BC) & 13 \\
\hline Cyathicula coronata (Bull. ex Mérat) De Not. \& P. Karst. & Edo. Méx. (BC) & 7 \\
\hline Daldinia grande Child & B.C. (BE) & 36 \\
\hline Dasyscyphus brasiliensis (Mont.) Le Gal & Mor., Ver. (BM) & 7 \\
\hline D. cerinus (Pers.) Fuckel & Edo. Méx. (BC) & 7 \\
\hline D. corticola (Massee) Dennis & Edo. Méx. (BM) & 7 \\
\hline D. orinocensis (Pat. \& Gail) Dennis & Hgo. (BC) & 9 \\
\hline D. virgineus Gray & Coah., Edo. Méx. & 7 \\
\hline - Diaporthe macrospora Wehmeyer & Pue. (BC) & 59 \\
\hline Diatrype albopruinosa (Schwein.) Cooke & Ver. (BM) & 10 \\
\hline D. capnostoma Berk. \& Ravenel & Qro. (BM) & 10 \\
\hline D. tumida Ellis \& Everh. & Coah. (BE) & 10 \\
\hline Diatrypella verrucaeformis var. spegazziniana Sacc. & Col. (VT) & 10 \\
\hline Elaphomyces muricatus Fr. & N.L. (BE, BPE) & 20 \\
\hline $\begin{array}{l}\text { Entoleuca mammata (Wahll.: Fr.) J. D. Rogers \& } \\
\text { Y. M. Ju } \\
\quad=\text { Hypoxylon mammatum (Wahll.) J. H. Mill. }\end{array}$ & Ver. (VT) & 40 \\
\hline - Entonaema globosum R. Heim & Camp., Oax. (VT) & 48,49 \\
\hline - E. liquescens Moell. & Tamps., Ver. (VT, BM) & 26,48 \\
\hline E. pallida G. Martin & Chis. (VT) & 36 \\
\hline Eutypa flavovirens (Pers.: Fr.) Tul. & Edo. Méx. (BC) & 10 \\
\hline Eutypella prunastri (Pers.: Fr.) Sacc. & N.L. $(B C)$ & 10 \\
\hline Fracchiaea heterogena Sacc. & Tamps. (VT) & 50 \\
\hline Genea arenaria Harkn. & N.L. (BE) & 5 \\
\hline G. hispidula Berk. ex Tul. & N.L. (BE) & 20 \\
\hline Geoglossum glabrum var. americanum Mains & Edo. Méx. (BC) & 7 \\
\hline G. glutinosum Pers.: Fr. & Dgo., Mor. (BC) & 7 \\
\hline G. nigritum (Fr.) Cooke & Edo. Méx. (BC) & 7 \\
\hline Geopyxis vulcanalis (Peck) Sacc. & Edo. Méx. (BC) & 9 \\
\hline Helvella albella Quél. & Edo. Méx., Ver. (BC) & 12 \\
\hline
\end{tabular}


Cuadro 1. Continuación.

\begin{tabular}{|c|c|c|}
\hline Especies & Entidades & Literatura \\
\hline H. corium (Weberb.) Massee & Mich. (BPE) & 12 \\
\hline H. ephippium Lév. & Son. (BPE) & 45 \\
\hline H. pezizoides Fr. & Mor., Son. (BE, BPE) & 2,45 \\
\hline H. queletii Bres. & Ver. (BM) & 12 \\
\hline H. stevesii Peck & Mor. (BM) & 2 \\
\hline H. villosa (Hedw. ex Kunth) Dissing \& Nannf. & Mich. (BPE) & 46 \\
\hline Humaria leucoloma (Hedw.) Quél. & Ver. (BM) & 12 \\
\hline Humarina permuda (Cooke) Seaver & Son. (VT) & 17 \\
\hline Hydnobolites cerebriformis Tul. \& C. Tul. & N.L. (BE) & 5 \\
\hline Hymenoscyphus fructigenus (Bull. ex Mérat) Gray & N.L. (BPE) & 7 \\
\hline H. herbarum (Pers.: Fr.) Dennis & N.L. $(B C)$ & 7 \\
\hline H. phyllogenus (Rehm) Kunth & Mor. (BPE) & 3 \\
\hline H. vitelinus (Rehm) Kunth & N.L. (BC) & 7 \\
\hline Hypocrea avellanea Rogersons \& Carey & Hgo. (BPE) & 43 \\
\hline H. citrina var. americana Canham & Hgo., Mor. (BC) & 7 \\
\hline Hypomyces chrysospermus Tul. & Ver. (BM) & 42 \\
\hline $\begin{array}{l}\text { H. lateritium (Fr.) Tul. } \\
\quad=\text { Peckiella lateritia (Fr.) Maire }\end{array}$ & $\begin{array}{l}\text { Edo. Méx., Gro., Jal., } \\
\text { Mor. (BC) }\end{array}$ & 42 \\
\hline H. luteovirens (Fr.) Tul. & Edo. Méx., Mor. (BM) & 42 \\
\hline - H. macrosporus Seaver & $\begin{array}{l}\text { D.F., Edo. Méx., Hgo., } \\
\text { Mich., Mor., Pue., Ver. } \\
\text { (VT) }\end{array}$ & 21,42 \\
\hline H. rosellus (Alb. \& Schwein.) Tul. & Oax. (VT) & 7 \\
\hline Hypoxylon aeruginosum J. H. Mill. & Q. Roo (VT) & 28 \\
\hline H. aeruginosum var. macrosporum J. D. Rogers & Ver. (BM) & 36 \\
\hline $\begin{array}{l}\text { H. anthochroum Berk. \& Broome } \\
\bullet=H \text {. albostigmatosum Speg. }\end{array}$ & $\begin{array}{l}\text { Nay., Sin., Q. Roo } \\
\text { (VT) }\end{array}$ & 28,31 \\
\hline H. archeri Berk. & $\begin{array}{l}\text { Chis., Hgo., Ver. (VT, } \\
\text { BM) }\end{array}$ & 35 \\
\hline H. bovei var. microspora J. H. Mill. & Ver. (VT) & 40 \\
\hline - H. crocopeplum Berk. \& M. A. Curtis & Nay., Q. Roo, Tamps. & 28,31 \\
\hline H. dieckmanii Theiss. & Oax. (BM) & 28 \\
\hline H. duranii J. D. Rogers & Yuc. (VT) & 28 \\
\hline H. erythrostroma J. H. Mill. & Tamps., Ver. (BM, VT) & 28 \\
\hline H. fendleri Berk. ex Cooke & Camp., Q. Roo (VT) & 28 \\
\hline H. fragiforme (Pers.: Fr.) Kickx & Ver. (VT) & 40 \\
\hline H. fuscum Pers.: Fr. & Chis. (BM) & 40 \\
\hline - H. haematostroma Mont. & Nay., Q. Roo, Sin., & 28,31 \\
\hline & Son., Ver. (VT) & 45 \\
\hline H. howeianum Peck & Coah. (BC) & 28 \\
\hline H. hypomyltum Pers.: Fr. & Q. Roo, Tab. (VT) & 22,28 \\
\hline H. hypophaleum (Berk. \& Ravenel) J. H. Mill. & Tamps. (VT) & 35 \\
\hline H. investiens (Schwein.) M. A. Curtis & Tab. (VT) & 28 \\
\hline H. jecorinum Berk. \& Ravenel & Hgo., N.L. (BM, VT) & 28,35 \\
\hline $\begin{array}{l}\text { H. lenormandii (Berk. \& M. A. Curtis) Sacc. } \\
\bullet \quad=H \text {. oodes Berk. \& Broome }\end{array}$ & $\begin{array}{l}\text { Chis., Nay., N.L., Sin. } \\
\text { (VT) }\end{array}$ & 28,31 \\
\hline
\end{tabular}


Cuadro 1. Continuación.

\begin{tabular}{|c|c|c|}
\hline Especies & Entidades & Literatura \\
\hline$=H$. riograndense $\mathrm{Rehm}$ & & \\
\hline H. lividipigmentum San Martín, Y. M. Ju \& J. D. Rogers & Oax., Q. Roo (VT) & 28 \\
\hline H. michelianum Ces. \& De Not. & $\begin{array}{l}\text { Edo. Méx., N.L. (VT, } \\
\text { BC) }\end{array}$ & 40 \\
\hline H. microplacum (Berk. \& M. A. Curtis) J. H. Mill. & Oax. (VT) & 40 \\
\hline H. monticulosum Mont. & Q. Roo (VT) & 28 \\
\hline H. moriforme Henn. & $\begin{array}{l}\text { Chis., Tamps., Q. Roo } \\
\text { (BM, VT) }\end{array}$ & 28 \\
\hline $\begin{array}{l}\text { H. mulleri J. H. Mill. } \\
\quad=H \text {. placentiforme Berk. \& M. A. Curtis }\end{array}$ & $\begin{array}{l}\text { Col., Oax., Tamps., } \\
\text { Ver. (BM, VT) }\end{array}$ & 28,40 \\
\hline H. multiforme Fr. & Edo. Méx. (BC) & 35 \\
\hline H. nitens (Ces.) Y. M. Ju \& J. D. Rogers & Chis. (VT) & 28 \\
\hline H. notatum Berk. \& M. A. Curtis & Ver. (BM) & 35 \\
\hline H. nummularium var. australe (Cooke ) J. H. Mill. & Oax., Pue. (VT, BM) & 40 \\
\hline H. nummularium var. rumpens (Cooke) J. H. Mill. & Ver. (VT) & 40 \\
\hline H. nummularium var. theissenii (Syd.) J. H. Mill. & Col. (VT) & 35 \\
\hline H. olivicolor San Martín, Y. M. Ju \& J. D. Rogers & Camp. (VT) & 28 \\
\hline - H. papillatum Ellis \& Everh. & sin loc. & 39 \\
\hline H. perforatum (Schwein.: Fr.) Fr. & N.L. (VT) & 28 \\
\hline H. placentiforme Berk. \& M. A. Curtis & Camp., Tamps. (VT) & 28 \\
\hline H. polyporum (Starb.) Y. M. Ju & Camp., Tamps. (VT) & 28 \\
\hline H. pseudostipitatum Y. M. Ju \& J. D. Rogers & Q. Roo (VT) & 28 \\
\hline H. purpureonitens Y. M. Ju \& J. D. Rogers & Chis. (VT) & 28 \\
\hline H. rickii Y. M. Ju \& J. D. Rogers & Oax. (VT) & 28 \\
\hline H. rosellinioides Hehn. & Tamps. (VT) & 57 \\
\hline H. rubigineo-areolatum Rehm & $\begin{array}{l}\text { Tamps., Ver., Yuc. } \\
\text { (VT) }\end{array}$ & 35 \\
\hline H. rubiginosum var. rubigineum (G. H. Otth) J. H. Mill. & D.F., Jal. (BC) & 40 \\
\hline H. rubiginosum var. tropica J. H. Mill. & Jal. (VT) & 24 \\
\hline - H. sclerophaeum Berk. \& M. A. Curtis & Nay., sin loc. & 31,39 \\
\hline H. subgilvum Berk. \& Broome & Tamps. (BM) & 28 \\
\hline H. subrutilum Starb. & Tamps. (BM) & 28 \\
\hline H. symphyon A. Möller & Oax. (VT) & 29 \\
\hline H. thouarsianum var. gilletianum (Sacc.) J. H. Mill. & $\begin{array}{l}\text { Col., Chis., Edo. Méx., } \\
\text { Jal., N.L., Oax., Tamps., } \\
\text { Ver. (BC, VT) }\end{array}$ & 35,40 \\
\hline $\begin{array}{l}\text { H. thouarsianum var. macrosporum San Martín, } \\
\text { Y. M. Ju \& J. D. Rogers }\end{array}$ & Chis. (VT) & 28 \\
\hline - H. truncatum (Schwein.: Fr.) J. H. Mill. & $\begin{array}{l}\sin \text { loc., N.L., Q. Roo } \\
\text { (VT) }\end{array}$ & 28,39 \\
\hline Hysterium angustatum Alb. \& Schwein. ex Mérat & Q. Roo (VT) & 21 \\
\hline $\begin{array}{l}\text { lodowynnea auriformis (Pat. ex Le Gal) Medel, Guzmán } \\
\quad \& \text { S. Chacón }\end{array}$ & Ver. (BM) & 38 \\
\hline Kotlabaea aff. deformis (P. Karst.) Svreck & Edo. Méx. (BC) & 9 \\
\hline Kretschmariella culmorum (Cooke) Y. M. Ju \& J. D. & Qro. (BM) & 35 \\
\hline
\end{tabular}


Cuadro 1. Continuación.

\begin{tabular}{|c|c|c|}
\hline Especies & Entidades & Literatura \\
\hline \multicolumn{3}{|l|}{$=$ Hypoxylon culmorum Cooke } \\
\hline Kretzchmaria cf. bulgarioides Rehm & N.L. (BE) & 53 \\
\hline K. aff. cetrarioides (Welw. \& Curr.) Sacc. & Tamps. (VT) & 53 \\
\hline K. heliscus (Mont.) Massee & Chis., Ver. (VT) & 36,53 \\
\hline K. micropus (Fr.) Sacc. & Oax. (VT) & 53 \\
\hline K. cf. mauritania (Durieu \& Mont.) Pat. & Oax. (VT) & 53 \\
\hline Lamprospora nigrans (Morgan) Seaver & Mor. (VT) & 2 \\
\hline $\begin{array}{l}\text { Leprieuria bacillum (Mont.) Laessøe J. D. Rogers \& } \\
\quad \text { Whalley }\end{array}$ & Chis., Ver. (VT) & 29,53 \\
\hline Lopadostoma turgidum (Pers.: Fr.) Traverso & Tamps. (BE) & 29 \\
\hline Melastiza chateri (Smith) Boud. & Mich., Ver. (BC) & 11 \\
\hline Melogramma bulliardii Tul. \& C. Tul. & Ver. (BM) & 33 \\
\hline Microglossum olivaceum (Pers.) Gillet & Edo. Méx. (BM) & 16 \\
\hline M. viride (Schradr.) Gillet & Mich. (BC) & 16 \\
\hline Morchella guatemalensis Guzmán, M. F. Torres \& Logem. & Jal. (BPE) & 25 \\
\hline M. umbrina Boud. & Ver. (BPE) & 23 \\
\hline $\begin{array}{l}\text { Mycopepon smithii var. mexicanum San Martín y J. D. } \\
\text { Rogers }\end{array}$ & Chis. (VT) & 51 \\
\hline Nectria cinnabarina (Tode: Fr.) Fr. & $\begin{array}{l}\text { D.F., Edo. Méx., Hgo., } \\
\text { Ver. (BM, BC) }\end{array}$ & 7 \\
\hline N. peziza (Tode: Fr.) Fr. & Ver. (BM) & 22 \\
\hline $\begin{array}{l}\text { Nemania bipapillata (Berk. \& M. A. Curtis) Pouzar } \\
\quad=\text { Hypoxylon subannulatum Henn. \& E. Nym. }\end{array}$ & Qro. (BM) & 35 \\
\hline $\begin{array}{l}\text { N. confluens (Tode: Fr.) Laessøe \& Spooner } \\
=\text { Hypoxylon confluens (Tode: Fr.) C. West. }\end{array}$ & Tamps. (VT) & 35 \\
\hline N. effusa Nitschke & Tamps. (BM) & 57 \\
\hline Nitschkia grevillei (Rehm) Nannf. & Ver. (BM) & 33 \\
\hline Orbilia cruenta (Schwein.) Seaver & Yuc. (VT) & 22 \\
\hline Otidea grandis Pers. & Hgo. (BPE) & 18 \\
\hline O. leporina var. minor (Rehm) Sacc. & Hgo. (BE) & 11 \\
\hline O. smithii Kanouse & Hgo. (BE) & 46 \\
\hline Pachyphloeus citrinus Berk. \& Broome & N.L. (BE) & 20 \\
\hline P. virescens Gilkey & N.L. (BE) & 20 \\
\hline Patinellaria cubensis (Berk. \& M. A. Curt.) Dennis & Ver. (BM) & 34 \\
\hline Penzigia conostoma (Mont.) J. H. Mill. & Ver. (BM) & 8 \\
\hline P. enteroleuca (Speg.) J. H. Mill. & Ver. (BM) & 8 \\
\hline Peroneutypa heterocantha (Sacc.) Berl. & D.F., Mor. (VT) & 41 \\
\hline Peziza abietina Pers. & Mor., Son. (BE) & 14,17 \\
\hline P. bruneoatra Desm. & Son. (BE) & 17 \\
\hline P. micheli (Boud.) Dennis & Gro. (BE) & 46 \\
\hline P. pustulata (Hedw.) Pers. & Gro. (BPE) & 46 \\
\hline P. succosa Berk. & Mor. (BPE) & 2 \\
\hline P. sylvestris Boud. & Mor. (BPE) & 2 \\
\hline Pezizella chrysostigma (Fr.) Sacc. & D.F. (BC) & 7 \\
\hline Phillipsia gigantea Seav. & Pue. & 32 \\
\hline P. aff. hartmanii (Phillips \& Cooke) Rifai & Q. Roo (VT) & 21 \\
\hline
\end{tabular}


Cuadro 1. Continuación.

\begin{tabular}{|c|c|c|}
\hline Especies & Entidades & Literatura \\
\hline Plectania campylospora (Berk.) Nannf. \& Korf & Gro. (BM) & 46 \\
\hline P. melastoma (Sow.: Gray) Fuckel & Edo. Méx., Gro. (BC) & 46 \\
\hline Pocillum cesatii (Mont.) De Not. & Ver. (BM) & 12 \\
\hline Podosordaria leporina (Ellis \& Everh.) Dennis & Ver. (BM) & 12 \\
\hline Pseudoplectania nigrella (Pers.: Fr.) Fuckel & $\begin{array}{l}\text { Edo. Méx., Gro. (BM, } \\
\text { BE) }\end{array}$ & 46 \\
\hline P. vogesiaca (Pers.) Seaver & Gro., Mich. (BM, BC) & 46 \\
\hline Rosellinia aquila (Fr.: Fr.) De Not. & N.L. (BE) & 55 \\
\hline R. corticium (Schwein.: Fr.) Sacc. & N.L. (BE) & 55 \\
\hline R. evansii Laessøe \& Spooner & Tamps. (BPE) & 57 \\
\hline R. necatrix Prilleux & Chis., Oax. (VT) & 55 \\
\hline R. procera Syd. \& P. Syd. & Chis., Oax. (VT) & 55 \\
\hline R. subiculata (Schwein.: Fr.) Sacc. & Ver. (BPE) & 55 \\
\hline R. sublimata (Durieu \& Mont.) Pass. \& Thüm. & $\begin{array}{l}\text { Chis., Q. Roo, Tamps. } \\
\text { (VT) }\end{array}$ & 55 \\
\hline - Saccobolus thaxterii Brumm. & Edo. Méx. & 30 \\
\hline $\begin{array}{l}\text { Sarcoscypha occidentalis (Sowerby) Sacc. } \\
\quad=\text { Plectania occidentalis (Schwein.) Seaver }\end{array}$ & Hgo., Mor. (BM, BPE) & 2,6 \\
\hline Schizoxylon pruiniferum Sherwood & Ver. (VT) & 33 \\
\hline Scortechinia acanthostroma Sacc. \& Berl. & Tamps., Ver. (VT) & 50 \\
\hline Scutellinia cubensis (Berk. \& M. A. Curt.) Seaver & Chis. (VT) & 33 \\
\hline S. diaboli (Velen.) Le Gal & Edo. Méx. (BE) & 46 \\
\hline S. umbrarum (Fr.) Lambotte & Son. (BPE) & 17 \\
\hline Stictis stellata Wallr. & D.F. (BC) & 9 \\
\hline Strossmayeria basitricha (Sacc.) Dennis & Oax. & 27 \\
\hline Thamnomyces chordalis Fr. & Chis. (VT) & 55 \\
\hline Thaxteria didyma (Speg.) Sacc. & Chis. (VT) & 50 \\
\hline $\begin{array}{l}\text { Thuemenella cubispora (Ellis \& Holw.) Boedijn } \\
\quad=\text { Chromocreopsis cubispora (Ellis \& Holw.) Seaver }\end{array}$ & Mor. (BM) & 7 \\
\hline Trichobelonium obscurum (Rehm) Rehm & Mor. (BM) & 7 \\
\hline Trichoglossum hirsutum Mains var. hirsutum & N.L. (BC) & 7 \\
\hline T. variabile (Durand) Nannf. & Ver. (BC) & 7 \\
\hline T. velutipes (Peck) Durand & Mich., Ver. (BM, BC) & 1,15 \\
\hline T. walteri (Berk.) Durand & Mich. (BC) & 15 \\
\hline Tuber candidum Hawker & N.L. (BE) & 20 \\
\hline T. maculatum Vittad. & N.L. (BE) & 5 \\
\hline T. rufum var. nitidum (Vittad.) I. Fisch. & N.L., Tamps. (BE) & 5 \\
\hline T. separans Gilkey & N.L. (BE) & 5 \\
\hline Ustulina deusta (Hoffm.: Fr.) Petrak & Tamps. (VT, BM) & 57 \\
\hline Wynnea macrotis (Berk.) Berk. & Ver. (BM) & 37 \\
\hline Xylaria adscendens (Fr.) Fr. & $\begin{array}{l}\text { Tamps., Chis., Oax., } \\
\text { Q. Roo (BM, VT) }\end{array}$ & 52,56 \\
\hline$X$. aenea (Mont.) Fr. & Chis. (BM) & 56 \\
\hline X. alata San Martín \& J. D. Rogers & Tamps. (BM, VT) & 52,56 \\
\hline X. allantoidea (Berk.) Fr. & $\begin{array}{l}\text { Chis., Q. Roo, Ver. } \\
\text { (BM, VT) }\end{array}$ & 52,56 \\
\hline
\end{tabular}


Cuadro 1. Continuación.

\begin{tabular}{|c|c|c|}
\hline Especies & Entidades & Literatura \\
\hline X. amphithele San Martín \& J. D. Rogers & Tamps. (BM, VT) & 52,56 \\
\hline X. anisopleura (Mont.) Fr. & $\begin{array}{l}\text { Chis., Oax., Q. Roo, } \\
\text { Tab., Tamps., Ver. } \\
\text { (BM, VT) }\end{array}$ & 52,56 \\
\hline X. apiculata Cooke & Chis., Tamps. (BM) & 56 \\
\hline$X$. aristata Mont. & Tamps. (BM, VT) & 52,56 \\
\hline$X$. aff. bambooensii Lloyd & Q. Roo (VT) & 52 \\
\hline X. boergesenii (Fred. \& Winge) P. Cannon & Oax., Tab., Ver. (VT) & 56 \\
\hline$X$. brachiata Sacc. & $\begin{array}{l}\text { Camp., N.L., Oax., } \\
\text { Q. Roo, Tamps., Ver. } \\
\text { (BM, VT) }\end{array}$ & 52,56 \\
\hline X. brasiliensis (Theiss.) Lloyd & Edo. Méx. (BM) & 19 \\
\hline X. cf. brevipes Sacc. & N.L. (VT) & 52 \\
\hline$X$. ciliata Pat. & D.F. (BE) & 56 \\
\hline X. claviceps San Martín \& J. D. Rogers & $\begin{array}{l}\text { Camp., Chis., Oax. } \\
\text { (VT) }\end{array}$ & 52,56 \\
\hline$X$. aff. comosa (Mont.) Fr. & Chis. (VT) & 52 \\
\hline$X$. cf. corniculata Sacc. & Tamps. (BM) & 52 \\
\hline X. corniformis var. macrospora Bres. apud Theiss. & Chis., Q. Roo (BE) & 56 \\
\hline X. delicatula Starb. & Oax., Q. Roo (VT) & 56 \\
\hline$X$. aff. dichotoma (Mont.) Fr. & $\begin{array}{l}\text { Camp., N.L., Q. Roo } \\
\text { (BE, VT) }\end{array}$ & 52,56 \\
\hline$X$. aff. enterogena (Mont.) Fr. & $\begin{array}{l}\text { Hgo., N.L., Tamps. } \\
\text { (BM) }\end{array}$ & 52 \\
\hline X. enteroleuca (Speg.) P. Martín & $\begin{array}{l}\text { Chis., Jal., N.L., } \\
\text { Tamps., Ver. } \\
\text { (VT, BM, BE) }\end{array}$ & 56 \\
\hline X. entosulphurea J. D. Rogers, San Martín \& Y. M. Ju & $\begin{array}{l}\text { Mich., Mor., Tamps. } \\
\text { (BM, BE) }\end{array}$ & 49 \\
\hline X. frustulosa (Berk. \& M. A. Curtis) Cooke & Tamps. (VT) & 56 \\
\hline X. gracillima (Fr.) Fr. & $\begin{array}{l}\text { Camp., N.L., Q. Roo, } \\
\text { Tamps. (BM, VT) }\end{array}$ & 52,56 \\
\hline X. guazumae San Martín \& J. D. Rogers & Oax., Tamps. (BM, VT) & 52,56 \\
\hline X. hyperythra (Mont.) Fr. & Ver. (VT) & 56 \\
\hline$X$. ianthino-velutina (Mont.) Fr. & $\begin{array}{l}\text { Chis., N.L., Q. Roo, } \\
\text { Tamps., Ver. (VT) }\end{array}$ & 52,56 \\
\hline X. cf. inaequalis Berk. \& M. A. Curtis & Camp., Q. Roo (VT) & 52,56 \\
\hline X. juniperus var. asperula Starb. & $\begin{array}{l}\text { Camp., Chis., N.L., } \\
\text { Q. Roo, Tamps. (VT, } \\
\text { BE) }\end{array}$ & 56 \\
\hline X. juruensis Henn. & Q. Roo (VT) & 52 \\
\hline X. kegeliana (Lév.) Fr. & Oax., Tamps. (BM, VT) & 52,56 \\
\hline$X$. laevis Lloyd & Chis., Q. Roo (VT) & 56 \\
\hline$X$. cf. laevis Lloyd & $\begin{array}{l}\text { Chis., Q. Roo, Tamps. } \\
\text { (VT) }\end{array}$ & 52,56 \\
\hline
\end{tabular}


Cuadro 1. Continuación.

\begin{tabular}{|c|c|c|}
\hline Especies & Entidades & Literatura \\
\hline X. lancea Lloyd & $\begin{array}{l}\text { Oax., Q. Roo, Tab., } \\
\text { Tamps. }\end{array}$ & 56 \\
\hline$X$. cf. longiana Rhem & $\begin{array}{l}\text { D.F., Jal., N.L., Oax., } \\
\text { Tamps. (BPE, VT) }\end{array}$ & 52,56 \\
\hline X. longipes var. tropica San Martín \& J. D. Rogers & Chis. (VT) & 52,56 \\
\hline X. luxurians (Rehm) Lloyd & Chis. (VT) & 56 \\
\hline X. magniannulata San Martín \& J. D. Rogers & Q. Roo (VT) & 52 \\
\hline X. magnoliae J. D. Rogers & Tamps. (BM) & 26 \\
\hline X. maitlandii (Dennis) D. Hawksw. & Chis. (VT) & 52 \\
\hline X. melanura (Lév.) Sacc. & Chis. (BM) & 56 \\
\hline X. cf. mellisii var. nuda (Dennis) D. Hawksw. & Tamps. (BM, VT) & 52,56 \\
\hline$X$. cf. microceras (Mont.) Fr. & $\begin{array}{l}\text { Camp., Chis., Tamps. } \\
\text { (BM) }\end{array}$ & 52,56 \\
\hline X. muscula Lloyd & $\begin{array}{l}\text { Q. Roo, Tamps. (BM, } \\
\text { VT) }\end{array}$ & 52 \\
\hline X. mussoriensis Dargan & Q. Roo, Tamps. (VT) & 56 \\
\hline$X$. cf. nigrescens (Sacc.) Lloyd & Chis., Oax., Ver. (VT) & 52,56 \\
\hline X. oxyacanthe Tul. & Tamps., Ver. (BM, VT) & 52,56 \\
\hline$X$. pallide-ostiolate Henn. & Q. Roo $(\mathrm{BM}, \mathrm{VT})$ & 52,56 \\
\hline X. persicaria (Schwein.: Fr.) Berk. \& M. A. Curtis & Tamps., Ver. (BM) & 52,56 \\
\hline X. phosphorea Berk. & Tamps. (BM) & 56 \\
\hline X. phyllocharis Mont. & Chis., Q. Roo (VT) & 52,56 \\
\hline X. poitei (Lév.) Fr. & $\begin{array}{l}\text { Jal., N.L., Oax., Q. Roo, } \\
\text { Tamps., Ver. (VT) }\end{array}$ & 52,56 \\
\hline X. rickii (Theiss.) P. Martín & Camp. (VT) & 56 \\
\hline X. rizhomorpha (Mont.) Mont. & Chis., Q. Roo (VT) & 56 \\
\hline X. rythidophloea Mont. & Chis., Ver. (VT) & 56 \\
\hline X. scabriclavula San Martín \& J. D. Rogers & Q. Roo (VT) & 52 \\
\hline X. schweinitzii Berk. \& M. A. Curtis & Chis., Ver. (VT) & 52,56 \\
\hline X. squamulosa San Martín \& J. D. Rogers & Q. Roo (VT) & 52,56 \\
\hline$X$. striata Pat. & Jal., Tamps. (VT) & 56 \\
\hline X. uniapiculata San Martín \& J. D. Rogers & $\begin{array}{l}\text { Camp., Chis., Oax., } \\
\text { Q. Roo, Tamps. (BM, } \\
\text { VT) }\end{array}$ & 52,56 \\
\hline
\end{tabular}

* Las especies señaladas con • no fueron consideradas por García-Romero et al. (1970) y Chacón y Guzmán (1983c), en sus respectivos períodos. Para el nombre completo de las entidades federativas véase el Cuadro 5 y para las referencias bibliográficas de la columna de Literatura, consúltese el Cuadro 2. Las abreviaciones entre paréntesis a continuación de las entidades federativas indican: VT vegetación tropical, BM bosque mesófilo de montaña, BC bosque de coníferas, BPE bosque de pinoencino y BE bosque de encino. 
Cuadro 2. Referencias bibliográficas citadas en el Cuadro 1.

\begin{tabular}{|c|c|c|c|}
\hline & Bandala et al., 1989 & & Kobayasi, 1978 \\
\hline 2. & Bautista et al., 1986 & 31. & Martin, 1969 \\
\hline 3. & Bautista et al., 1992 & 32. & Martínez-Alfaro et al., 1983 \\
\hline 4. & Carrión y Chacón, 1993 & 33. & Medel y Chacón, 1988a \\
\hline 5. & Cázares et al., 1992 & 34. & Medel y Chacón, 1988b \\
\hline 6. & Chacón y Bautista, 1988 & 35. & Medel et al., 1989 \\
\hline 7. & Chacón y Guzmán, 1983b & 36. & Medel y Chacón, 1992 \\
\hline 8. & Chacón y Guzmán, 1983c & 37. & Medel et al., 1995 \\
\hline 9. & Chacón y Guzmán, 1985 & 38. & Medel et al., 1996 \\
\hline 10. & Chacón y Medel, 1988 & 39. & Miller, 1961 \\
\hline 11. & Chacón y Medel, 1990 & 40. & Pérez-Silva, 1983 \\
\hline 12. & Chacón y Medel, 1992 & 41. & Pérez-Silva, 1986 \\
\hline 13. & Cifuentes et al., 1990 & 42. & Pérez-Silva et al., 1983a \\
\hline 14. & Cifuentes et al., 1993 & 43. & Pérez-Silva et al., 1983b \\
\hline 15. & Díaz-Barriga, 1988 & 44. & Pérez-Silva et al., 1985 \\
\hline 16. & Díaz-Barriga y Chacón, 1992 & 45. & Pérez-Silva et al., 1996 \\
\hline 17. & Esqueda Valle et al., 1992 & 46. & Pompa-González y Cifuentes, 1991 \\
\hline 18. & Frutis y Guzmán, 1983 & 47. & Rodríguez et al., 1993 \\
\hline 19. & Frutis et al., 1985 & 48. & Rogers, 1981 \\
\hline 20. & Garza et al., 1985 & 49. & Rogers et al., 1996 \\
\hline 21. & Guzmán, 1977 & 50. & San Martín, 1996a \\
\hline 22. & Guzmán, 1983 & 51. & San Martín, 1996b \\
\hline 23. & Guzmán et al., 1995 & 52. & San Martín y Rogers, 1989 \\
\hline 24. & Guzmán-Dávalos et al., 1983 & 53. & San Martín y Rogers, 1993a \\
\hline 25. & $\begin{array}{l}\text { Guzmán-Dávalos y Rodríguez- Alcantar, } \\
1993\end{array}$ & $\begin{array}{l}54 . \\
55 .\end{array}$ & $\begin{array}{l}\text { San Martín y Rogers, } 1993 b \\
\text { San Martín y Rogers, 1995a }\end{array}$ \\
\hline 26. & Heredia, 1989 & 56. & San Martín y Rogers, 1995b \\
\hline 27. & Iturriaga y Korf, 1990 & 57. & San Martín y Rogers, 1996 \\
\hline 28 & Ju, Y.-M y D. Rogers, 1996 & 58. & Valenzuela, 1990 \\
\hline 20 & $M$ et & 5 & e, 1961 \\
\hline
\end{tabular}

\section{DISCUSION}

Son 544 las especies de ascomicetos macroscópicos hasta ahora citadas de México, 269 del período de 1983-1996 y 275 desde el siglo pasado hasta 1982. Dicho total corresponde a alrededor de $9 \%$ de todos los hongos registrados en el país, que según Guzmán (1998) suman aproximadamente 6 000. A nivel mundial los ascomicetos conocidos representan menos de $2 \%$ del gran total que existe en la Tierra, que según Hawksworth (1991) es de más de 1500000 especies. Esto demuestra lo mucho que falta por estudiar y lo que seguramente se está perdiendo debido al alto deterioro del medio y de la vegetación, en especial la de los bosques tropicales (Guzmán, 1998), cuya pérdida anual/ mundial es de 20 millones de hectáreas al año (Halffter, 1992). Guzmán (1998) estimó que sólamente se conoce $3.5 \%$ de las 200000 especies de hongos que crecen en México y que las áreas tropicales son las más diversas, pero las menos exploradas. 
A pesar de que los bosques templados (BC, BPE y BE) son los más intensamente muestreados desde el punto de vista micológico en el país, en la presente lista (Cuadro 1) se encontró que la mayoría de las especies se han citado de vegetación tropical y subtropical (Cuadro 4) (señaladas como VT y BM en el Cuadro 1). Sugiere ello que es allí en donde potencialmente se encontraría un gran número de especies, tal como lo observaron Guzmán et al. $(1995,1997)$ con respecto a los hongos mexicanos en general y a los de Veracruz en particular, y Hawksworth $(1991,1993)$ a nivel mundial. Acorde con lo anterior, Whalley (1993) advirtió que $75 \%$ de los Xylariales habita en los trópicos y es interesante constatar que este grupo es el mejor representado en México, con 243 especies (Cuadro 3).

Cuadro 3. Números de especies de diferentes órdenes de ascomicetos macroscópicos citadas de México en tres distintas épocas

\begin{tabular}{|l|c|c|r|r|}
\hline & Hasta 1970 & Hasta 1983* & Hasta 1996 & Total \\
\hline Diaporthales & & 1 & 1 & 2 \\
Diatrypales & & 25 & 7 & 7 \\
Dothideales & & 2 & 1 & 28 \\
Elaphomycetales & 2 & 13 & 36 & 5 \\
Helotiales & 7 & 14 & 10 & 56 \\
Hypocreales & 6 & 49 & 2 & 30 \\
Ostropales & 32 & 39 & 5 & 2 \\
Pezizales & 19 & 66 & 158 & 127 \\
Sordariales & & 209 & 269 & 243 \\
Xylariales (= Sphaeriales) & 66 & & 544 \\
\hline Total & & & \\
\hline
\end{tabular}

* Aquí se incluyen las 14 especies omitidas.

Cuadro 4. Distribución por tipo de vegetación de las especies registradas en este trabajo.

\begin{tabular}{|l|c|}
\hline Vegetación & Especies citadas \\
\hline Vegetación tropical & 137 \\
Bosque mesófilo de montaña & 81 \\
Bosque de coníferas & 40 \\
Bosque de encino & 33 \\
Bosque de pino-encino & 23 \\
\hline
\end{tabular}

Referente a la distribución conocida de los ascomicetos en las entidades federativas del país, ésta se muestra en el Cuadro 5, en donde se incluyen todas las especies desde el primer listado de 1970. Dicha información está claramente relacionada con las 
exploraciones que se han llevado a cabo en el país y con la ubicación de los centros de investigación y/o enseñanza. Llama la atención la falta de muestreos en entidades como Colima, Nayarit, Sinaloa, Tabasco y Yucatán que poseen vegetación tropical. Resulta interesante observar que se tienen muy pocos registros de ascomicetos de las zonas áridas y semiáridas, enclavadas en gran parte de las entidades federativas del noroeste del país. Los trabajos de Esqueda-Valle et al. (1992) y Pérez-Silva et al. (1996) parecen contener las pocas referencias sobre tales hongos de regiones de clima seco.

Cuadro 5. Total de especies de ascomicetos citadas por entidad federativa*

\begin{tabular}{|lr|lr|}
\hline Entidad & Total & Entidad & Total \\
\hline (Ags.) Aguascalientes & 0 & (Mor.) Morelos & 60 \\
(B.C.) Baja California & 1 & (Nay.) Nayarit & 10 \\
(B.C.S.) Baja California Sur & 0 & (N.L.) Nuevo León & 73 \\
(Camp.) Campeche & 24 & (Oax.) Oaxaca & 65 \\
(Coah.) Coahuila & 7 & (Pue.) Puebla & 33 \\
(Col.) Colima & 6 & (Qro.) Querétaro & 5 \\
(Chih.) Chihuahua & 12 & (Q. Roo) Quintana Roo & 59 \\
(Chis.) Chiapas & 84 & (S.L.P.) San Luis Potosí & 27 \\
(D.F.) Distrito Federal & 28 & (Sin.) Sinaloa & 10 \\
(Dgo.) Durango & 34 & (Son.) Sonora & 46 \\
(Edo. Méx.) Estado de México & 69 & (Tab.) Tabasco & 20 \\
(Gro.) Guerrero & 37 & (Tamps.) Tamaulipas & 87 \\
(Gto.) Guanajuato & 1 & (Tlax.) Tlaxcala & 11 \\
(Hgo.) Hidalgo & 51 & (Ver.) Veracruz & 135 \\
(Jal.) Jalisco & 76 & (Yuc.) Yucatán & 10 \\
(Mich.) Michoacán & 54 & (Zac.) Zacatecas & 5 \\
\hline
\end{tabular}

* Se incluyen en esta relación todos los registros desde el siglo pasado hasta el presente.

Con respecto a la conservación de estos organismos en la naturaleza, existen varias especies que de acuerdo con nuestras observaciones, ya es muy raro encontrarlas. Tal es el caso de algunos Pezizales y Xylariales de zonas tropicales y subtropicales, o bien, de los Helotiales y Pezizales asociados al bosque mesófilo de montaña; a pesar de ello, sólo cinco especies de Morchella (dos amenazadas y tres bajo protección especial) están consideradas en el listado de especies en peligro de extinción publicado en el Diario Oficial de la Federación (1994). Finalmente, es notable hacer ver que el estudio de la diversidad de los ascomicetos en México, como el de todos los macromicetos, ha tenido un desarrollo significativo en los últimos años, como lo discutió Guzmán (1998) recientemente. Esto se basa en el hecho de que entre 1983 y 1996 se registraron 269 especies de ascomicetos macroscópicos, mientras que desde el siglo pasado hasta 1982 únicamente lo fueron 275. 


\section{AGRADECIMIENTOS}

Los autores agradecen a las autoridades del Instituto de Ecología, A.C. las facilidades para la realización del presente trabajo y los apoyos recibidos por el CONACYT y CONABIO en diversas épocas. Se reconoce también la colaboración de Ma. Eugenia Ramírez, Fidel Tapia, Florencia Ramírez-Guillén y Juan Lara, todos del Instituto de Ecología, A.C.

\section{LITERATURA CITADA}

Bandala, V. M., G. Guzmán y L. Montoya. 1989. Algunos Geoglossaceae (Fungi, Ascomycotina, Helotiales) poco conocidos de México. Rev. Mex. Mic. 5: 117-123.

Bautista, N., S. Chacón y G. Guzmán. 1986. Ascomycetes poco conocidos de México, III. Especies del estado de Morelos. Rev. Mex. Mic. 2: 85-104.

Bautista, N., V. M. Mora y L. Acosta-Urdapilleta. 1992. Ascomycetes poco conocidos de México, V. Especies de Morelos, parte 3. Int. J. Mycol. \& Lichenol. 5: 195-198.

Carrión, G. y S. Chacón. 1993. Primer registro de Balansia cyperi (fungi) en México. Rev. Mex. Mic. 9: $165-167$.

Cázares, E., J. García, J. Castillo y J. Trappe. 1992. Hypogeous fungi from Northern Mexico. Mycologia 84: 341-359.

Chacón, S. y N. Bautista. 1988. Ascomycetes poco conocidos en México, IV. Especies de Morelos, parte 2. Biotica 13: 35-40.

Chacón, S. y G. Guzmán. 1983a. Especies de macromicetos citadas de México. V. Ascomycetes parte II. Bol. Soc. Méx. Mic. 18: 103-114.

Chacón, S. y G. Guzmán. 1983b. Ascomycetes poco conocidos de México. Bol. Soc. Mex. Mic. 18: 183-218.

Chacón, S. y G. Guzmán. 1983c. Penzigia conostoma y Penzigia enteroleuca (Ascomycetes, Pyrenomycetes, Sphaeriales) en México. Bol. Soc. Mex. Mic. 18: 29-32.

Chacón, S. y G. Guzmán. 1985. Ascomycetes poco conocidos de México. II. Discomycetes. Rev. Mex. Mic. 1: 131-134.

Chacón, S. y R. Medel. 1988. Ascomycetes lignícolas de México I. Diatrypales. Rev. Mex. Mic. 4: 323-331.

Chacón, S. y R. Medel. 1990. Ascomycetes poco conocidos en México. V. Descripción de algunos Pezizales. Rev. Mex. Mic. 6: 199-206.

Chacón, S. y R. Medel. 1992. Ascomycetes poco conocidos de México VI. Algunos Discomycetes y Pyrenomycetes. Rev. Mex. Mic. 8: 55-62.

Cifuentes, J., M. Villegas, L. Pérez-Ramírez, M. Bulnes, V. Corona, G. Ma. del R. González, I. Jiménez, A. Pompa y G. Vargas. 1990. Observaciones sobre la distribución, hábitat e importancia de los hongos de Los Azufres, Michoacán. Rev. Mex. Mic. 6: 133-149.

Cifuentes J., M. Villegas-Ríos y L. Pérez-Ramírez. 1993. Hongos macroscópicos. In: Luna-Vega, I. y J. Llorente (ed.). Historia natural del parque ecológico estatal Omiltemi, Chilpancingo, Gro. México. Universidad Autónoma de México y Comisión Nacional para el Conocimiento y Uso de la Biodiversidad. México, D.F. pp. 59-126.

Diario Oficial de la Federación. 1994. Organo del Gobierno Federal de México, Norma Oficial Mexicana NOM-059-ECOL-1994. México, D.F. Vol. 488 (10) 1.

Díaz-Barriga, H. 1988. Primer registro de Trichoglossum velutipes (Geoglossaceae, Ascomycetes) para México. Acta Bot. Mex. 2: 1-4.

Díaz-Barriga, H. y S. Chacón. 1992. Nuevo registro del género Microglossum (Ascomycotina, Geoglossaceae) para la micoflora mexicana. Acta Bot. Mex. 20: 5-8. 
Esqueda-Valle, M., E. Pérez-Silva y M. Coronado-Andrade. 1992. Nuevos registros de Pezizales para Sonora. Rev. Mex. Mic. 8: 43-54.

Frutis, I. y G. Guzmán. 1983. Contribución al conocimiento de los hongos del estado de Hidalgo. Bol. Soc. Mex. Mic. 18: 219-266.

Frutis, I., R. E. Chio y A. Estrada-Torres. 1985. Nuevos registros de macromicetos del estado de México. Rev. Mex. Mic. 1: 285-300.

García-Romero, L., G. Guzmán y T. Herrera. 1970. Especies de macromicetos citadas de México I. Ascomicetes, Tremellales y Aphyllophorales. Bol. Soc. Mex. Mic. 4: 54-76.

Garza, F., J. García y J. Castillo. 1985. Macromicetos asociados al bosque de Quercus rysophylla en algunas localidades del centro del estado de Nuevo León. Rev. Mex. Mic. 1: 423-438.

Guzmán, G. 1977. Identificación de los hongos. Limusa. México, D.F. 452 pp.

Guzmán, G. 1983. Los hongos de la Península de Yucatán. II. Nuevas exploraciones y adiciones micológicas. Biotica 8: 71-100.

Guzmán, G. 1998. Inventorying the fungi of Mexico. Biodiversity and Conservation 7: 369-384.

Guzmán, G., V. M. Bandala y L. Montoya. 1997. An overview on the tropical fungi from Mexico. In: Janardhanan, K. K., C. Rajendran, K. Natarajan y D. L. Hawksworth (eds.). Tropical mycology. Science Publ. Inc., Enfield. pp. 115-148.

Guzmán, G., V. M. Bandala, L. Montoya, S. Chacón y G. Carrión (en prensa). Los hongos. Introducción a la micobiota. In: Llorente, J. (ed.). Biodiversidad de Veracruz. Consejo Nacional para el Conocimiento y Uso de la Biodiversidad, México, D.F.

Guzmán-Dávalos, L., G. Nieves y G. Guzmán. 1983. Hongos del estado de Jalisco II. Especies depositadas en el herbario ENCB. Bol. Soc. Mex. Mic. 18: 125-158.

Guzmán-Dávalos, L. y O. Rodríguez-Alcantar. 1993. Registro de Morchella guatemalensis (Fungi, Ascomycotina) para México. Bol. Inst. Bot. Univ. Guadalajara 1: 471-475.

Halffter, G. 1992. Diversidad biológica y cambio global. Ciencia y Desarrollo 18: 33-38.

Hawksworth, D. L. 1991. The fungal dimension of biodiversity: magnitude, significance, and conservation. Mycol. Research 95: 641-655.

Hawksworth, D. L. 1993. The tropical fungal biota: census, pertinence, prophylaxis, and prognosis. In: Isaac, S., J. C. Frankland, R. Watling y A. J. S. Whalley (eds.). Aspects of tropical mycology. Cambridge University Press. Melbourne. pp. 265-294.

Hawksworth, D. L., P. M. Kirk, B. C. Sutton y D. N. Pegler. 1995. Ainworth \& Bisby s dictionary of the Fungi. 8a. ed. IMI, CAB. Surrey. 616 pp.

Heredia, G. 1989. Estudio de los hongos de la Reserva de la Biosfera El Cielo, Tamaulipas. Consideraciones sobre la distribución y ecología de algunas especies. Acta Bot. Mex. 7: 118.

Iturriaga, T. y R. P. Korf. 1990. A monograph of the discomycete genus Strossmayeria (Leotiaceae) with comments on its anamorphs Pseudospiropes (Dematiaceae). Mycotaxon 36: 383-454.

Ju, Y.-M. y J. D. Rogers. 1996. A revision of the genus Hypoxylon. Mycologia Memoirs 20, APS Press. Minesota. 365 pp.

Ju, Y.-M., F. San Martín y J. D. Rogers. 1993. Three xylariaceous fungi with scolecosporous conidia. Mycotaxon 47: 219-228.

Kobayasi, Y. 1978. Mycological survey of Mexican volcano Popocatepetl (2). J. Jap. Bot. 54: 86-94.

Martin, P. 1969. Studies in the Xylariaceae V. Euhypoxylon. Jour. South Afr. Bot. 35: 149-206.

Martínez-Alfaro, M. A., E. Pérez-Silva y E. Aguirre-Acosta. 1983. Etnomicología y exploraciones micológicas en la Sierra Norte de Puebla. Bol. Soc. Mex. Mic. 18: 51-64.

Medel, R. y S. Chacón. 1988a. Ascomycetes lignícolas de México, II. Algunos Pyrenomycetes y Discomycetes. Mic. Neotrop. Aplic. 1: 87-96.

Medel, R. y S. Chacón. 1988b. Primer registro en México de Patinellaria cubensis (Discomycetes, Helotiales) y su asociación con Xylaria arbuscula. Rev. Mex. Mic. 4: 9-12.

Medel, R., S. Chacón y G. Guzmán. 1989. Especies conocidas y nuevos registros de Hypoxylon (Sphaeriales, Xylariaceae) en México. Rev. Mex. Mic. 5: 149-168. 
Medel, R. y S. Chacón. 1992. Ascomycetes lignícolas de México, III. Algunos Sphaeriales. Int. J. Mycol. \& Lichenol. 5: 253-260.

Medel, R., G. Guzmán y S. Chacón. 1995. New data on the genus Wynnea in Mexico. Mycotaxon 55: 295-299.

Medel R., G. Guzmán, S. Chacón y R. P. Korf. 1996. lodowynnea, a new genus of the Pezizales known from Africa and tropical America. Mycotaxon 59: 127-135.

Miller, J. H. 1961. A monograph of the world species of Hypoxylon. University of Georgia Press. Athens, Ga. $158 \mathrm{pp}$.

Pérez-Silva, E. 1983. Distribución de algunas especies del género Hypoxylon (Pyrenomycetes) en México. An. Inst. Biol. UNAM, Ser. Bot. 54: 1-22.

Pérez-Silva, E. 1986. Presencia del género Peroneutypa en la micoflora mexicana. An. Inst. Biol. UNAM, Ser. Bot. 57: 11-16.

Pérez-Silva, E. Aguirre-Acosta y T. Herrera. 1983a. Distribución e importancia de algunas especies de Hypomyces (Hypocreales) en México. An. Inst. Biol. UNAM, Ser. Bot. 54: 203-218.

Pérez-Silva, E., E. Aguirre-Acosta y T. Herrera. 1983b. Descripción y nuevos registros de hongos micoparásitos de México. Bol. Soc. Mex. Mic. 18: 71-84.

Pérez-Silva, E., J. A. Samaniego-Gaxiola y S. Perrusquia-Jasso. 1985. Hongos fimícolas de México, IV. Primer registro del género Ascodesmis (Pezizales, Ascomycetes). Rev. Mex. Mic. 1: 239242.

Perez-Silva, E., M. Esqueda-Valle y A. Armenta-Calderón. 1996. Ascomycetes de Sonora I: Discomycetes y Pyrenomycetes. Rev. Mex. Mic. 12: 97-106.

Pompa-González, A. y J. Cifuentes. 1991. Estudio taxonómico de los Pezizales de los estados de Guerrero, Hidalgo, Estado de México y Michoacán. Rev. Mex. Mic. 7: 87-112.

Rodríguez, O., L. Guzmán-Dávalos y L. S. Vásquez. 1993. Nuevos registros de hongos para México. Micol. Neotrop. Apl. 6: 61-71.

Rogers, J. D. 1981. Sarcoxylon and Entonaema (Xylariaceae). Mycologia 73: 28-69.

Rogers, J. D., F. San Martín y Y.-M. Ju. 1996. Mexican fungi: Xylaria entosulphurea sp. nov. and neotypification of Entonaema globosum. Mycotaxon 58: 483-487.

San Martín, F. 1996a. Contribución al conocimiento de cinco géneros de la familia Nitschkiaceae (Hymenoascomycetes, Sordariales). Acta Bot. Mex. 36: 43-52.

San Martín, F. 1996b. Una nueva variedad de Mycopepon smithii (Ascomycetes, Pleosporales). Acta Bot. Mex. 35: 9-12.

San Martín, F. y J. D. Rogers. 1989. A preliminary account of Xylaria of Mexico. Mycotaxon 34: 283374.

San Martín, F. y J. D. Rogers. 1993a. Kretzchmaria, Leprieuria and Poronia in Mexico. Mycotaxon 48: $174-191$.

San Martín, F. y J. D. Rogers. 1993b. Biscogniauxia and Camillea in Mexico. Mycotaxon 47: 229-258.

San Martín, F. y J. D. Rogers. 1995a. Rosellinia and Thamnomyces in Mexico. Mycotaxon 53: 115127.

San Martín, F. y J. D. Rogers. 1995b. Notas sobre la historia y relaciones de hospedante y distribución del género Xylaria (Pyrenomycetes, Sphaeriales) en México. Acta Bot. Mex. 30: 21-40.

San Martín, F. y J. D. Rogers. 1996. A preliminary list of Xylariaceous fungi at El Cielo Biosphere Reserve, in Tamaulipas, Mexico. Acta Bot. Mex. 37: 23-31.

Valenzuela, R., 1990. El género Chlorociboria en México. Rev. Mex. Mic. 6: 125-131.

Welden, A. L y P. A. Lemke. 1961. Notas sobre algunos hongos mexicanos. Bol. Soc. Bot. Mex. 26: $1-24$.

Whalley, A. J. S. 1993. Tropical Xylariaceae. Their distribution and ecological characteristic. In: Isaac, S., J. C. Frankland, R. Watling y A. J. S. Whalley (eds.). Aspects of tropical mycology. Cambridge University Press. Cambridge. pp. 103-120. 